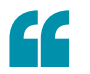

anticancer therapies that target RNAs seem to be one step closer to being a new class of anticancer drug

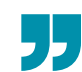

\title{
THERAPY
}

\section{A big step for targeting RNAs}

There are many unanswered questions about the development of drugs that target RNAs. For example, will such drugs accumulate to a sufficient extent in tumour cells to produce a therapeutic effect? What RNAs are good targets? Can we selectively target tumour cells with systemically administered drugs? Will there be significant long-term side effects? This month, two papers report findings that take us closer to answering some of these questions.

Mark Davis and colleagues report the initial findings of a Phase I clinical trial of systemic administration of CALAA-01 in patients with solid tumours that are refractory to standard therapy. CALAA-01 is a nanoparticle drug containing small interfering RNAs targeted to ribonucleoside reductase M2 ( $\underline{R R M 2})$. In addition, on its surface the

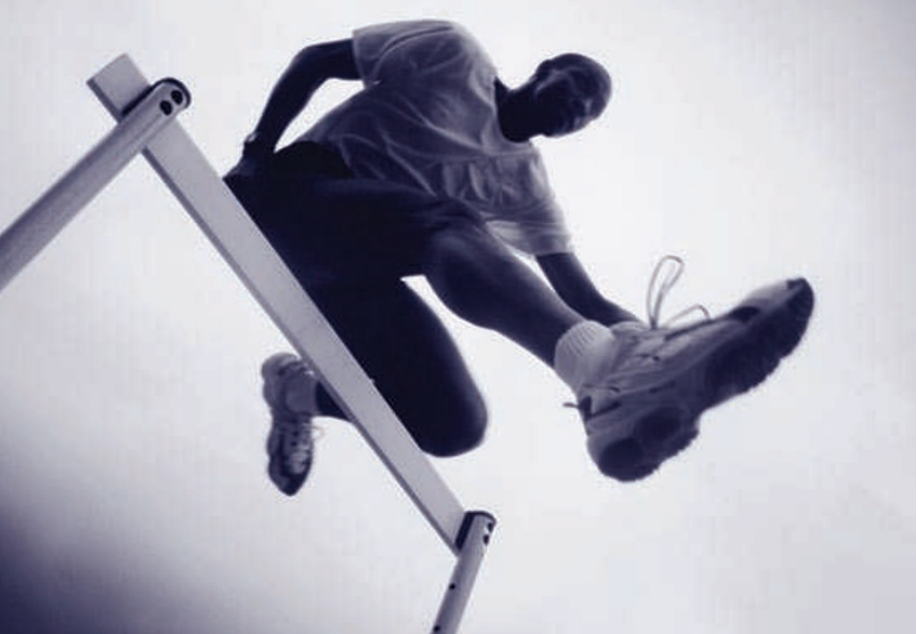

nanoparticle displays transferrin, which is a ligand for transferrin receptors that are often upregulated on tumour cells. The authors took tumour biopsy samples from three patients with metastatic melanoma, and each patient received a different dose of CALAA-01. They showed that the nanoparticles localized in the tumour cells - but not the surrounding normal epidermis - in a dose-dependent manner, and that $R R M 2$ mRNA and protein levels were reduced. They also found evidence of RNA interference pathway activation in biopsy samples from one patient. Although the pharmacodynamics of this drug are still to be fully characterized, the biopsy analyses suggest that the RNA interference effects could last several weeks.

So, can other types of RNA be therapeutically silenced in tumours? Li Ma and colleagues report the effects of systemic administration of antagomir-10b, an antisense oligonucleotide that silences the microRNA miR-10b. $m i R-10 b$ is induced by the transcription factor TWIST1 and targets $\mathrm{HOXD} 10$ mRNA, which consequently increases the expression of RHOC. The expression of miR-10b has been associated with high-grade tumours and metastases, particularly in breast carcinoma, pancreatic adenocarcinoma and glioblastoma.

Orthotopic transplantation of 4T1 mouse mammary tumour cells, which express high levels of TWIST1 and miR-10b, in syngeneic mice produces primary tumours that metastasize to the lungs. The authors found that systemic administration of antagomir-10b had little effect on the size and growth of the primary tumour but significantly reduced the number of metastases. Moreover, the authors found that HOXD10 levels increased in primary tumours from mice treated with antagomir-10b, but levels of other microRNAs (including miR-10a, which differs from miR-10b by one nucleotide) were unaffected. Silencing of miR-10b in $4 \mathrm{~T} 1$ cells prior to orthotopic injection also significantly decreased the number of metastases (primary tumour growth remained unaffected) indicating that the antimetastatic effects of antagomir-10b occur in the tumour cells rather than the tumour microenvironment. They also found that treatment with antagomir-10b did not suppress metastatic tumour growth when $4 \mathrm{~T} 1$ cells were injected in the tail vein, which bypasses the invasion and intravasation steps of metastasis. Finally, given that antagomir-10b was systemically administered the authors assessed the toxicity to normal mice treated with the same dosing schedule of antagomir-10b; they found no substantial effects.

Therefore, anticancer therapies that target RNAs seem to be one step closer to being a new class of anticancer drug.

Gemma K. Alderton

ORIGINAL RESEARCH PAPERS Davis, M. E. et al. Evidence of RNAi in humans from systemically administered siRNA via targeted nanoparticles. Nature 21 Mar 2010 (doi:10.1038/nature08956)| $\mathrm{Ma}$, L. et al. Therapeutic silencing of miR-10b inhibits metastasis in a mouse mammary tumor model. Nature Biotech. 28 Mar 2010 (doi:10.1038/ nbt.1618) 